\title{
GENÈSE ET DÉVELOPPEMENT DU PRINCIPE DE MOINDRE ACTION
} Première partie : de Fermat (1655) à Lagrange (1756)

\author{
Abdelmadjid MESLI \\ Aix-Marseille Université, CNRS, \\ IM2NP UMR7334, \\ 13397 Marseille Cedex 20, France \\ abdelmadjid.mesli@im2np.fr
}

\begin{abstract}
L'objet de la science moderne est de tracer dans le chaos et le flux de phénomènes une structure logique permettant d'interpréter et de transcender l'expérience. Depuis Galilée, la physique s'est construite à partir de quelques concepts fondamentaux dans lesquels les notions de temps et d'espace en constituent le cadre.
\end{abstract}

$\mathrm{D}$ epuis Fermat, et sa loi du temps minimum dans le trajet optique, un nouveau concept est apparu mettant en exergue la notion d'optimisation, à partir duquel le concept de «moindre action» s'est peu à peu imposé comme un élément fondateur de la structuration logique citée plus haut. Alors que la mécanique newtonienne et la théorie de la relativité se sont toutes deux construites sans le concept de moindre action, celui-ci les a redéfinies, aboutissant par extension à la formulation de la mécanique quantique. C'est l'évolution conceptuelle et historique du développement du principe de moindre action qui est décrite dans cet article, en retraçant l'histoire qui démarre avec Fermat et l'optique géométrique, trouvant son apogée avec Schrödinger et son équation, et se poursuivant avec Feynman et sa théorie de l'électrodynamique quantique. La première partie de cet article, qui en compte deux, traite de la période allant de Fermat à Lagrange se concentrant sur l'apport du principe à la mécanique classique. La deuxième partie discutera de l'apport décisif de Hamilton constituant la transition vers l'ère quantique et couvrant la période allant de 1900 et l'avènement du quantum d'action à Feynman.

\section{Introduction}

Les premières interrogations sur la nature, bien que fondées sur des expériences primitives, nous ont très vite imposé un certain nombre de concepts fondamentaux tels que temps, espace ou matière. Si le philosophe n'a jamais cessé de se questionner sur le contenu de tels concepts, le physicien, qu'il soit classique ou moderne, à travers ses deux piliers que sont la physique quantique et la physique relativiste, a pour motivation unique de poser une méthodologie capable de mener à une construction scientifique sur laquelle des déductions seraient possibles. Pour cela, il ne se pose pas la question du type "qu'est-ce que le temps, l'espace ou la matière?", car comme le précise Klein, définir une chose, c'est la ramener à une autre plus fondamentale [1]. Or, un concept, une fois défini comme fondamental, ne peut être ramené qu'à lui-même, d'où la difficulté. Le physicien s'attache plutôt à trouver ce qui relie les concepts de temps, d'espace et de matière, cette dernière caractérisée par sa masse [2], dès lors que ces trois entités s'imposent comme seuls moyens de rendre la nature intelligible.

Jusqu'à l'avènement, au début du vingtième siècle, de la théorie de la relativité, les trois concepts cités ci-dessus étaient séparés et absolus, et la matière se conservait. Einstein a joint les concepts de temps et d'espace en un seul concept espace-temps et établi l'équivalence masse-énergie avec la conséquence immédiate que l'on ne parle plus de conservation de la masse mais de celle de l'énergie [2], qui englobe de fait les deux entités. Pendant à peu près la même période, la physique quantique a mis un terme aux notions de trajectoire et de localisation d'une particule microscopique rendant impossible le fait de l'imaginer occuper un lieu précis avec une impulsion précise, conformément au principe d'incertitude de Heisenberg.

Un principe, établi à l'époque de la physique classique newtonienne, donc bien antérieur à ces deux révolutions scientifiques majeures, a non seulement survécu à ces deux nouvelles visions du monde, mais a également permis de les reformuler avec une surprenante élégance. Mieux encore, il a servi à développer l'électrodynamique quantique. Ce principe 
est celui de moindre action dont cet article fait l'objet. En langage moderne, il se définit comme la minimisation du transfert d'énergie entre les deux réservoirs d'énergie, que sont les énergies cinétique et potentielle, et ceci, dans l'intervalle de temps dévolu au déplacement entre deux points A et B. L'aspect fascinant de ce principe ne vient pas seulement du fait qu'il ait traversé le temps. Aussi étonnant que cela puisse paraitre, il a été développé à une époque où le mysticisme, la croyance irrationnelle ou la conviction religieuse faisaient partie intégrante de la vie des scientifiques et étaient librement reconnus. Déjà à l'époque de Galilée, on considérait que «le monde est divin parce qu'il est harmonieux", et plus on découvrait des lois physiques déchiffrant cette harmonie, plus on était persuadé de l'action divine. La foi religieuse était ainsi un «ingrédient» essentiel dans les réflexions de nombreux scientifiques, et pas des moindres, comme il sera mentionné dans cet article. Selon Galilée, la nature agit selon le plus simple des processus d'où sa conviction que la plus simple des relations lie temps, distance et vitesse. On peut voir poindre dans une telle affirmation le principe de moindre action que Fermat, contemporain de Galilée, appellera le «principe d'économie naturelle», avant que Maupertuis ne l'érige en principe de moindre quantité d'action.

Une fois dépouillé du contenu religieux, ce principe s'est révélé être d'une incomparable fécondité. Ce qui rend en effet certains principes importants, et celui de la moindre action en est un, c'est leur récurrence dans un grand nombre de descriptions, souvent dans des régions très éloignées du contexte de leur formulation initiale. Aucun concept ni principe n'a évidemment de caractère absolu et n'a par conséquent de sens, que par rapport à son devenir et ce que des générations de scientifiques en font au cours du temps. Le principe de moindre action a conduit de proche en proche à une série de reformulations de la mécanique classique par Euler et Lagrange, aboutissant à l'établissement par Hamilton de l'équivalent mécanique de l'optique ondulatoire. Dans cette même période d'effervescence scientifique, la théorie de la relativité a été reformulée sur la base du principe de moindre action, de même que la théorie électromagnétique de Maxwell.

On voit donc que la science ne progresse pas uniquement par l'accumulation de nouveaux faits mais également par l'approfondissement continu de fructueux concepts [3].

Le présent article, qui se compose de deux parties, est organisé comme suit. La section à suivre est dédiée à la chronologie et aux rappels historiques des principaux acteurs scientifiques qui ont pris part à la genèse, au développement ainsi qu'à la généralisation du principe de moindre action, en se limitant à l'ère pré-quantique. Le principe est traité en partant des conclusions de Fermat sur le trajet réfléchi ou diffracté d'un rayon de lumière en optique géométrique, ce dernier processus conduisant à la dichotomie entre moindre temps et moindre distance. Ensuite, vient la proposition de Maupertuis d'introduire d'abord le principe de moindre quantité d'action, qui prend clairement sa source dans les travaux de Fermat, avant de devenir simplement le principe de moindre action. Entrent alors en scène Euler et Lagrange qui ont posé les fondements de ce principe en établissant le puissant calcul variationnel. Nous verrons alors le lien entre l'action mécanique et l'action en optique géométrique.

La deuxième partie de l'article (à paraittre dans le prochain numéro de Photoniques) commence par le travail monumental de Hamilton et de Jacob Jacobi, clôturant la période pré-quantique et ouvrant la voie à l'avènement de la mécanique quantique. L'ère quantique démarre avec Max Planck et sa découverte du quantum d'action suivi par la concrétisation par Louis De Broglie, de l'idée de Hamilton, prédisant un lien entre la mécanique et l'optique ondulatoire. Erwin Schrödinger étend le travail de De Broglie en établissant son équation ondulatoire de la matière. Enfin, l'ultime étape de l'article décrira comment l'histoire prend un tournant étrange quand Richard Feynman reconsidère le concept de Fermat sur la base du photon et non plus sur celle de l'onde de lumière. Feynman déclare qu'en allant d'un point $\mathrm{A}$ à un point $\mathrm{B}$, le photon emprunte tous les chemins possibles, c'est-à-dire une infinité, un processus dans lequel l'approche de Fermat se réduit à une simple affaire de probabilité...

\section{Chronologie et repères historiques pré-quantiques du principe de moindre action}

La figure 1 montre l'échelle temporelle le long de laquelle s'inscrivent les principaux acteurs qui ont pris une part fondamentale au développement du principe de moindre action. Le choix de l'époque galiléenne comme origine de la genèse du principe de moindre action est tout à fait arbitraire. S'il fallait trouver une justification à ce choix, ce serait simplement du fait que Galilée est considéré comme le père de la science moderne. Il est, par ailleurs, utile de rappeler que les travaux en optique de Fermat, qui sont précurseurs du principe de moindre action, trouvent eux-mêmes leurs origines dans la plus haute antiquité [4]. Enfin, la contribution indirecte de Galilée au principe de moindre action se trouve dans sa tentative de résoudre un problème qui s'avèrera être à l'origine du calcul variationnel comme moyen de formaliser le principe de moindre action. Ce sera le fameux problème

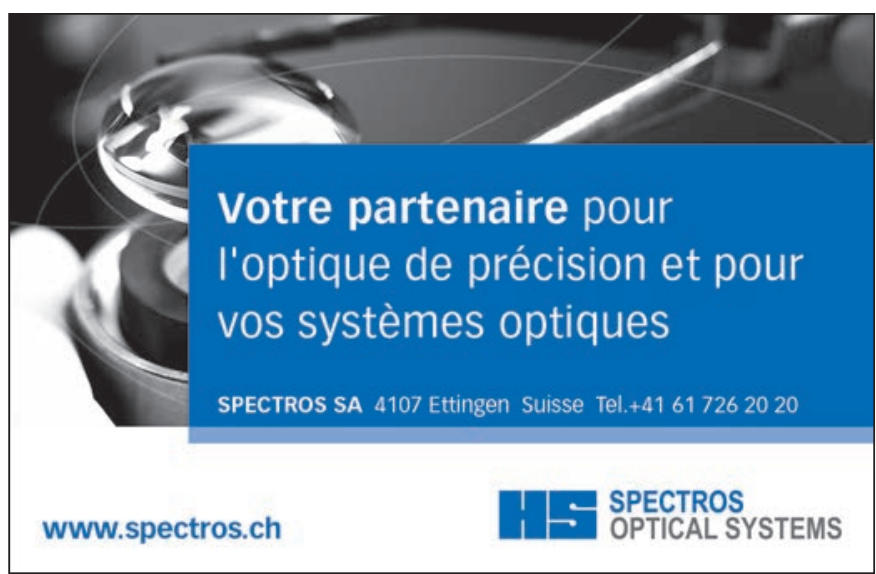



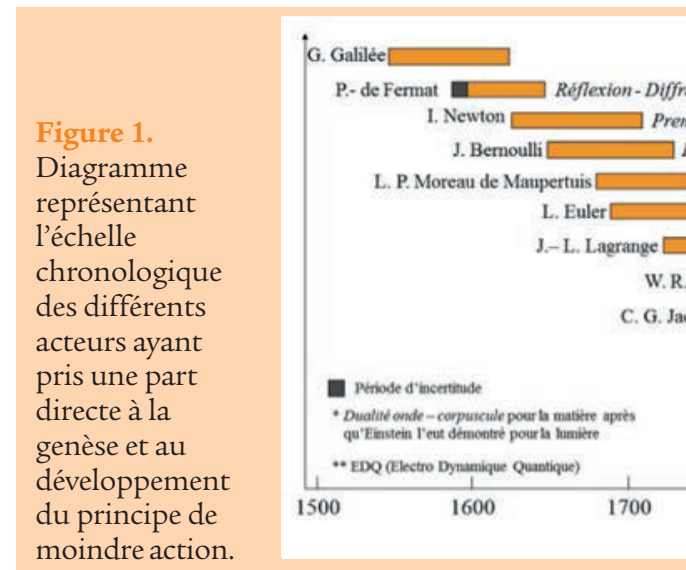

du brachistochrone (brachitos + chrone ou le temps le plus court), posé par J. Bernoulli à la communauté scientifique de l'époque, environ 50 ans après Galilée. Ce dernier part du traitement d'un corps se mouvant le long d'un plan incliné schématisée sur la figure 2. Après avoir établi la relation entre la distance parcourue le long du plan $\mathrm{AB}$ et le temps, la question suivante était de savoir s'il existait un autre chemin, marqué AMB pour lequel le trajet du corps est le plus rapide. Galilée propose à partir de son traitement du plan incliné que la courbe $\mathrm{AMB}$ est un arc de cercle, amenant à la conclusion que le brachistochrone est un cercle, ce qui s'avèrera faux. Galilée ne disposait pas d'une méthode adéquate pour traiter ce type de problème. Il faudra attendre Bernoulli.

\section{Pierre de Fermat}

Pierre de Fermat, contemporain de Galilée (voir figure 1), s'est intéressé au trajet de la lumière avec comme seule base de travail l'optique géométrique. Il pose le principe du temps minimum pour un rayon lumineux allant d'un point $A$ à un point $B$ se réfléchissant ou se réfractant sur une surface plane. Ce principe lui permet de retrouver le résultat, établi au premier siècle avant notre ère par Héron d'Alexandrie, que la réflexion de la lumière conduit à l'égalité des angles d'incidence et de réflexion. Par ailleurs, et contre Descartes et Newton, il démontre que la lumière, dont il ne connaissait pas la vitesse, va moins vite dans un milieu matériel que dans le vide. Il ne connaissait pas le concept d'indice de réfraction non plus mais considérait la «résistance» à la propagation de la lumière dans la matière. Le résultat est que l'angle de réfraction est plus faible que l'angle incident quand la lumière passe d'un milieu d'indice $\eta_{1}$ à un milieu d'indice $\eta_{2}$ avec $\eta_{1}<\eta_{2}$. Dans le langage de Fermat il faut remplacer cette inéquation par celle qui lie les vitesses, $u_{1}>u_{2}$, aboutissant au principe du trajet le plus rapide [5]. Ces deux effets sont illustrés à la figure 3 . Mathématiquement, et d'une manière générale, quand un rayon lumineux se propage dans un milieu d'indice de réfraction variable selon les trois dimensions $\eta(x, y, z)=c / u(x, y, z)$, le principe de moindre temps s'énonce par l'expression:

$\delta T=\delta\left(\int_{t_{A}}^{t_{B}} d t\right)=\delta\left(\int_{x_{A}}^{x_{B}} \frac{d l}{u(x, y, z)}\right)=\delta\left(\frac{1}{c} \int_{x_{A}}^{x_{B}} \eta(x, y, z) d l\right)=0$ où $T$ représente la durée de trajet du rayon lumineux, $d l$ est l'élément de déplacement entre les points A et $\mathrm{B}$ et $u(x, y, z)$ exprime la vitesse de la lumière dans un milieu d'indice de réfraction $\eta(x, y, z)$. La relation montre aisément que dans un milieu d'indice de réfraction constant le trajet de la lumière est rectiligne en accord avec l'optique géométrique. L'extension à deux milieux d'indice de réfraction différents mais constants, aboutit à la brisure de la trajectoire rectiligne à l'interface entre ces deux milieux et à la loi dite de Snell-Descartes. Fermat identifia son principe à celui d'un principe d'économie naturelle par lequel «la nature agit toujours par les moyens les plus aisés ou les plus économiques». On y aperçoit ici les prémisses de considérations mystiques qui s'affirmeront de plus en plus jusqu'à Maupertuis. L'équation (1) explique nombre de phénomènes comme celui du mirage illustré à la figure 4, du fait de la variation de l'indice de l'air $\eta(x, y, z)$ due à la chaleur émanant du sol, mais également celui de la déformation apparente du soleil au coucher par temps chaud.

\section{Isaac Newton}

Après cette parenthèse optique, qui s'avèrera être d'une incroyable richesse, car reprise par une génération de scientifiques visionnaires, esquissons l'idée majeure de la mécanique classique, initiée par Galilée et reprise par Newton, lequel a abordé le mouvement d'un corps grâce au calcul infinitésimal qu'il a, en parallèle avec Leibnitz, développé. Dans son approche, Newton fait le bilan des forces qui agissent sur le corps en mouvement à un instant et en un point donnés. Partant de cette condition initiale, il calcule la position en un autre point infiniment proche, parvenant ainsi de proche en proche (par itération) à reconstituer la trajectoire complète. Cette manière de procéder l'a conduit à établir sa première loi sous forme d'une équation différentielle exprimée ci-dessous et qui, une fois résolue, prédit la position du corps à n'importe quel instant ultérieur pourvu que soient connues les position et vitesse initiales:

$$
\vec{F}=\frac{d m \vec{v}}{d t}
$$


Dans cette équation, $\vec{F}$ résulte du bilan de toutes les forces agissant sur le corps et $m \vec{v}=\vec{p}$ représente la quantité de mouvement, une grandeur liée aux propriétés inertielles du corps en question. La quantité de mouvement, initialement introduite par Descartes, en tant que scalaire, alors qu'elle s'avèrera être une quantité vectorielle, sera d'une importance centrale dans le développement du principe de moindre action proposé par Maupertuis.

\section{Jean Bernoulli}

En 1696 Jean Bernoulli établit un lien entre l’optique géométrique et la mécanique. On assistera alors à un tournant dans l'évolution des idées en physique. Maupertuis, qui établit le principe de moindre quantité d'action, pose également une base formelle au couplage optique - mécanique qui mènera à un deuxième tournant, lorsque William Rowan Hamilton prédira le lien entre l'optique ondulatoire et la future mécanique quantique. Ce point sera discuté dans la deuxième partie.

Jean Bernoulli trouve dans le principe du moindre temps de Fermat un moyen de résoudre un problème mécanique qui conduira au brachistochrone. Il réexamine en effet la trajectoire du corps allant du point A vers le point B de la figure 2, situés sur un plan vertical, soumis à la seule gravitation mais contraint de suivre la trajectoire $\mathrm{AMB}$, le problème abordé mais non résolu par Galilée. Sur la base du trajet lumineux traversant deux milieux d'indices différents (à l'époque: de densités différentes), Jean Bernoulli a l'ingénieuse idée de donner un sens à la contrainte en stratifiant l'espace en une multitude de couches, la vitesse du corps variant au passage d'une couche à la suivante [6]. Le parallèle concerne un milieu optique d'indice continument variable où, à l'évidence, la trajectoire de la lumière ne sera pas une droite mais une courbe (voir l'exemple de la figure 4). Dans un tel milieu, le rapport de deux indices différents pour la lumière est dans un rapport similaire de celui des vitesses lors de la chute du corps dans le cas de la mécanique comme l'illustre la figure 5 (a). Dans le cas du Brachistochrone, l'astuce de Bernoulli permet de montrer que la courbe sera un arc de cycloïde et non pas un arc de cercle comme l'avait suggéré Galilée 50 ans plus tôt. La cycloïde est visualisée à la figure 5 (b) par la trajectoire que suit un point fixe, pris sur la circonférence d'une roue quand celle-ci roule sans glisser sur un plan quelconque.

Par ce travail, Bernoulli peut être considéré comme étant le premier à avoir traité un problème mécanique en utilisant un principe découlant de l'optique géométrique, en l'occurrence le principe de Fermat. Mais il reviendra à Hamilton d'aller beaucoup plus loin dans la réflexion de ce couplage entre l'optique et la mécanique.

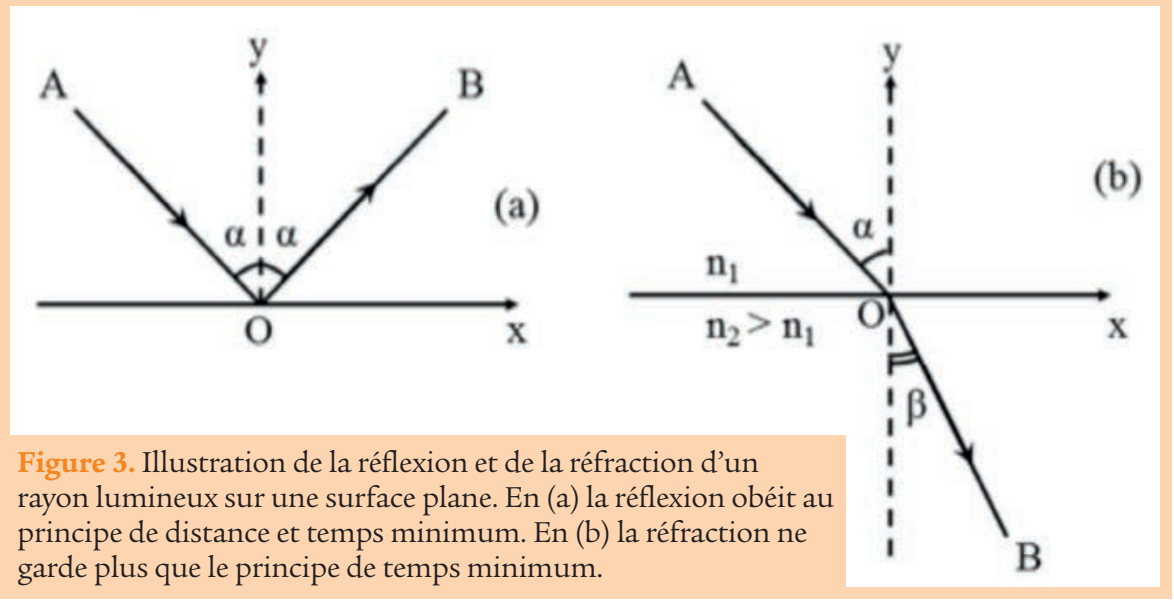

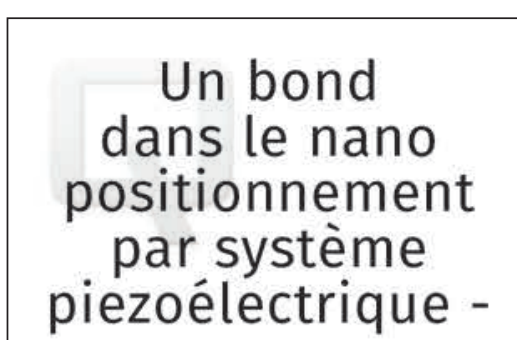
Lancement de la série
$\square$ d'Aerotech: nano-
positionneur piezo
électrique QNP et Piezo contrôleur QLAB

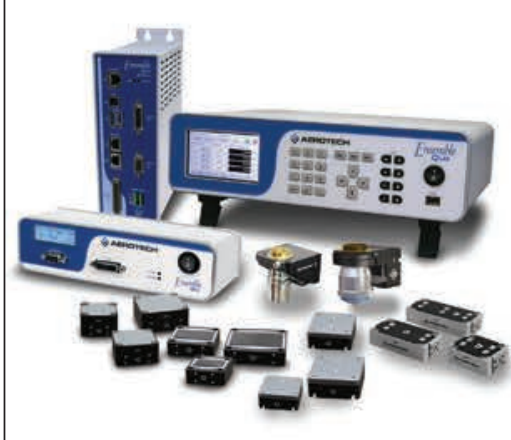

Les tables QNP présentent une raideur hors norme grâce à une fréquence de résonnance très élevée et une résolution sub-nanométrique. Elles sont donc idéales pour les applications pointues à faible encombrement telles que l'intérferometrie, la microscopie et les alignements d'extrême précision. Le contrôleur associé QLAB dispose d'un écran tactile et peut fonctionner de manière indépendante ou peut être connecté à un $\mathrm{PC}$ via Ethernet, ce qui le rend extrémement flexible dans toutes les situations. Avec des performances sub-nanométriques et un environnement de contrôle et de programmation très convivial, obtenir un positionnement nanométrique n'aura jamais été aussi facile.

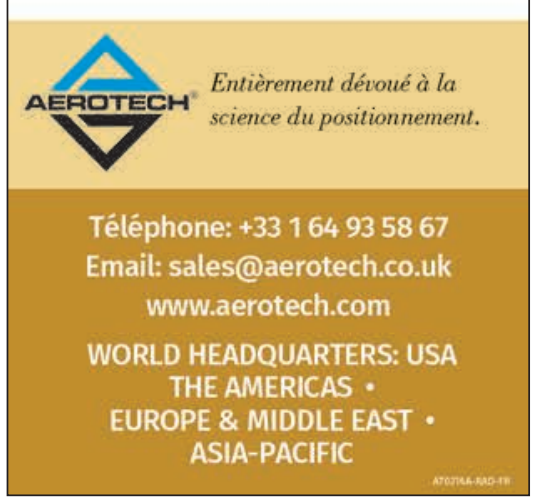




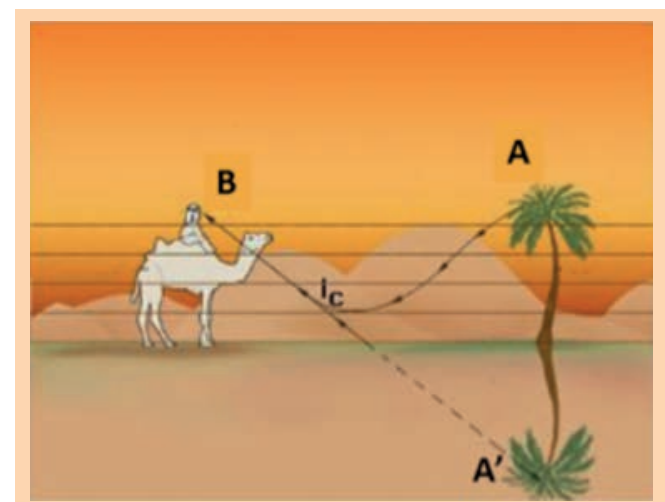

Figure 4. Illustration du mirage comme conséquence de la variation spatiale de l'indice de réfraction.

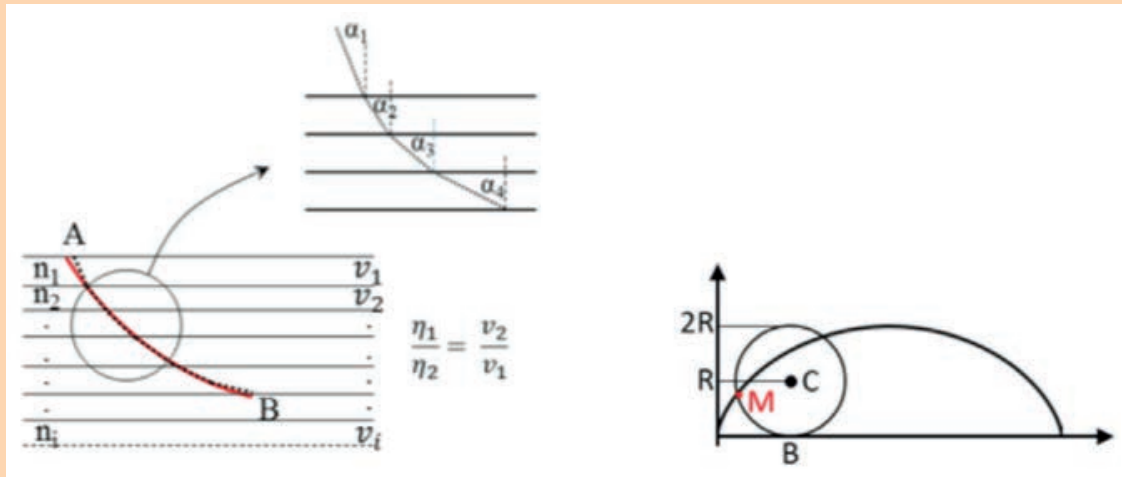

Figure 5. (a) Approche schématique utilisée par Jean Bernoulli pour traiter le problème du brachistochrone sur la base du travail entrepris en optique par Pierre de Fermat.

(b) La courbe du brachistochrone ou cycloïde issue du mouvement d'un point fixe $\mathrm{M}$ pris sur la circonférence d'une roue roulant sans glisser sur un plan.

\section{Pierre-Louis Moreau de Maupertuis}

Pierre-Louis Moreau de Maupertuis, qui était un ardent défenseur de la pensée newtonienne, proposa en 1744 d'étendre le principe d'économie du temps de Fermat au système mécanique. Ce sera le principe de moindre quantité d'action [7] qu'il définit comme étant égale à la circulation de la quantité de mouvement $\vec{p}=m \vec{v}$ le long d'une trajectoire dont l'élément de distance est $\overrightarrow{d l}$. Cette trajectoire débute au point $A$ et se termine au point $B$, et, la circulation devant être minimum, doit s'exprimer par

$$
\delta S=\delta\left(\int_{x_{A}}^{x_{B}} m \vec{v} d \vec{l}\right)=0
$$

Maupertuis, guidée par sa foi et sa profonde croyance, partagées par nombre de scientifiques célèbres de son époque, conclut en affirmant "Maintenant, voici ce principe, si sage, si digne de l'être suprême: lorsqu'il arrive quelque changement dans la Nature, la quantité d'Action employée pour ce changement est toujours la plus petite qu'il soit possible».

\section{Léonard Euler - Joseph-Louis Lagrange}

Le principe de Maupertuis, exprimé par la relation (3), trouve la même année (1744) une justification formelle dans le travail de Leonhard Euler et en 1756 Joseph-Louis Lagrange aboutit, dans le cas simple d'un mouvement à une dimension, à la définition moderne du principe de moindre action,

$$
\delta S=\delta\left(\int_{t_{A}}^{t_{B}} L(x, \dot{x}, t) d t\right)=0
$$

dans lequel la fonction à minimiser contient le lagrangien. Celui-ci exprime l'échange (ou la différence), au cours du trajet, entre deux réservoirs d'énergie que sont les énergies cinétique et potentiel du système considéré,

$$
L(x, \dot{x}, t)=T(\dot{x})-V(x)
$$

Remarquons le changement des bornes d'intégration ente les relations (3) et (4). Dans le cas d'un système conservatif, l'équivalence entre les relations (2), (3) et (4) est immédiate. Il suffit de remplacer dans l'équation (3) $\overrightarrow{d l}$ par $\vec{v} d t$ et procéder au changement approprié des bornes d'intégration. On perçoit ainsi dans ce principe une des caractéristiques des lois de la nature, qui se déduisent de principes d'optimisation régis par deux sources de mouvement. Dans l'exemple du système conservatif illustré par la figure 2, le réservoir d'énergie cinétique augmente aux dépends de celui de l'énergie potentielle dans une proportion optimale, seule responsable de la trajectoire réelle.

La différence majeure entre l'approche basée sur le principe de moindre action et celle de Newton est que ce dernier procède au calcul de la trajectoire de proche en proche en utilisant le calcul infinitésimal ou calcul différentiel menant à l'équation (2). Celle-ci sera résolue si on connaît la position et la vitesse du départ, le point d'arriver en découlera. Au contraire, la méthode d'Euler-Lagrange traite le mouvement dans sa globalité, et non par l'approche infinitésimale de Newton, partant des points de départ et d'arrivée, supposés connus. Le chemin réel sera celui qui minimise l'action.

Si le traitement mathématique de minimisation de l'équation (5) peut paraître plus complexe que celui de la résolution de l'équation différentielle de Newton, il permet néanmoins d'établir des lois fondamentales (dont celle de Newton) à partir de la seule connaissance du lagrangien du système.

Il est intéressant de noter que les deux formes équivalentes (3) et (4) conçoivent l'action comme variant dans l'espace mais également dans le temps. En effet, lors du mouvement, il convient de définir un point de départ $\left(x_{A}, t_{A}\right)$ et un point d'arrivée $\left(x_{B}, t_{B}\right)$ Entre ces deux coordonnées, seule une trajectoire est réelle, celle qui satisfait à la condition (3) ou (4). Toutes les autres sont considérées comme virtuelles. Dans le cas particulier d'un système dynamique où l'énergie se conserve, la comparaison se fait non pas entre tous les mouvements virtuels, mais seulement entre ceux qui satisfont à la loi de conservation de l'énergie. Dans ce cas, le point d'arrivée peut être 
laissé libre $(x, t)$. Nous verrons comment le formalisme de Hamilton-Jacobi exploite cette seconde approche, basée sur le rôle du temps, pour arriver à l'équivalence mécanique - optique ondulatoire, qui aboutira plusieurs années plus tard à l'établissement de l'équation de Schrödinger et la naissance de la mécanique quantique.

\section{Quel est le lien entre l'action en mécanique et l’action en optique géométrique?}

Nous avons vu que le principe de Fermat détermine non seulement la seule trajectoire possible, celle respectant le temps minimum, mais prédit également la courbure des rayons de lumière dans un espace à indice de réfraction continument variable comme le montre la relation (1), illustrée par la figure 4. L'action dans l'optique géométrique de Fermat est donc la durée du trajet entre les deux points A et B. Mais alors, quelle variable dans l'optique de Fermat joue le rôle de la variable temps dans l'action mécanique telle qu'elle est définie par la relation (4) ? La réponse à cette question équivaut à déterminer le «lagrangien optique».

En partant de la figure 3 et de la relation (1), il est très aisé d'exprimer le temps que le rayon lumineux met pour parcourir le trajet $A B$, selon qu'il s'agisse de réflexion ou de réfraction. En considérant le déplacement sur le plan $x \mathrm{O} y$, et, en remplaçant l'élément de déplacement $d l$ par $\sqrt{d x^{2}+d y^{2}}$ le temps est donné par

$$
T=\frac{1}{c} \int_{x_{A}}^{x_{B}} \eta(x, y) \sqrt{d x^{2}+d y^{2}}=\frac{1}{c} \int_{x_{A}}^{x_{B}} \eta(x, y) \sqrt{1+\bar{y}^{2}} d x
$$

où $y$ est une fonction de $x, \bar{y}$ exprime la dérivée par rapport à $x$ (le symbole $y$ étant réservé à la dérivée par rapport au temps). Ceci nous amène au lagrangien pour le trajet optique de Fermat s'exprimant par

$$
L_{\text {optique }}(y, \bar{y}, x)=\frac{1}{c} \eta(x, y) \sqrt{1+\bar{y}^{2}}
$$

dans lequel le rôle des différentes variables devient clair. Ainsi, dans l'optique de Fermat, la variable spatiale $x$ joue le rôle du temps défini dans le lagrangien mécanique alors que la variable $y$ garde la même signification dans les deux approches. Le principe de moindre action dans l'optique géométrique de Fermat peut donc s'écrire comme suit,

$$
\delta T=\delta\left(\int_{x_{A}}^{x_{B}} L_{\text {optique }}(y, \bar{y}, x) d x\right)=0
$$

d'où l'on déduit aisément que, dans le cas d'un milieu d'indice constant, la lumière suit un trajet rectiligne déjà mentionné plus haut. À partir de la même équation, on peut également montrer l'égalité des angles d'incidence et de réflexion ainsi que la loi de Snell-Descartes décrivant la réfraction. Le tableau ci-dessous compare les deux approches de Fermat et d'Euler-Lagrange.

\begin{tabular}{|c|c|}
\hline MÉCANIQUE: & OPTIQUE GÉOMÉTRIQUE: \\
EULER-LAGRANGE & FERMAT \\
\hline Action $S$ & durée $T$ \\
\hline Temps $t$ & Position horizontale $x$ \\
\hline Position $x$ & Position verticale $y$ \\
\hline Vitesse $\dot{x}$ & $\bar{y}=d t / d x$ pente dans le plan $x$ Oy \\
\hline lagrangien $L_{\text {mécanique }}(x, \dot{x}, t)$ & lagrangien $L_{\text {optique }}(y, \bar{y}, x)$ inverse de la vitesse \\
\hline
\end{tabular}

\section{small components MASSIVE IMPACT}

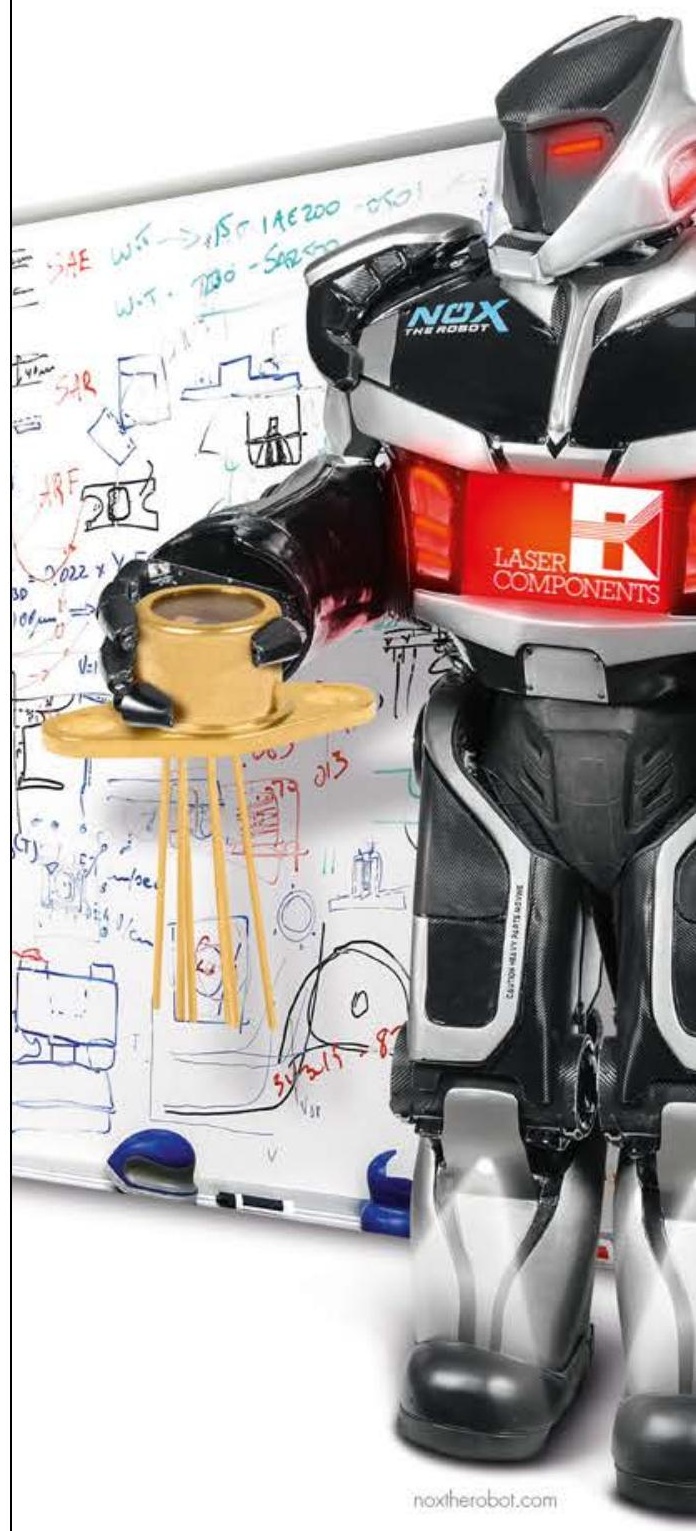

\section{We Manufacture IR Detectors}

- $(x)$ nnGaAs Photodiodes

- PbS \& PbSe Detectors

- Pyroelectric Detectors 
Le raisonnement établi ci-dessus peut être inversé, permettant de définir un indice de «réfraction mécanique» qui est responsable de la forme du trajet d'un corps soumis à un potentiel dépendant de l'espace à trois dimensions $V(x, y, z)$. Cet indice de réfraction, qui n'est autre que la quantité de mouvement, s'exprime par

$$
m v=\sqrt{2 m(E-V(x, y, z))}
$$

Et en remplaçant dans l'équation (3) on trouve l'expression,

$$
\delta S=\delta\left(\int_{x_{A}}^{x_{B}} \sqrt{2 m[E-V(x, y, z)]} d l\right)=0
$$

Cette équation est à comparer aux relations (3) et (8). Il est à noter que l'indice de réfraction optique est sans dimension, ce qui n'est pas le cas de l'indice de «réfraction mécanique». Comme on le voit, à l'instar du rayon lumineux se courbant dans un milieu d'indice $\eta(x, y, z)$, le potentiel $V(x, y, z)$ est une propriété de l'espace qui courbe la trajectoire d'un corps de masse m en mouvement.

\section{Quelle place pour le principe de moindire action en physique?}

Le caractère fondamental du principe de moindre action, et sa place dans la hiérarchie des principes et des lois en physique, continuent de susciter de profondes discussions, aussi bien philosophiques que scientifiques. Il convient par exemple de citer L. Boltzmann et R. Clausius qui ont perçu une relation entre ce principe et la seconde loi de la thermodynamique (augmentation de l'entropie pour tout système évoluant irréversiblement). Si la loi de conservation de l'énergie est requise dans l'évolution d'un système thermodynamique isolé, elle n'est pas suffisante. Entre deux directions d'évolution de ce système, toutes deux satisfaisant la loi de conservation de l'énergie, la seconde loi impliquant l'entropie décide laquelle est possible.

Le parallèle en mécanique est flagrant dans la mesure où le principe de conservation de l'énergie dérive du principe de moindre action et non l'inverse. Le premier est particulier alors que le deuxième est plus général. Un seul exemple suffit à illustrer le propos. Selon le principe de conservation d'énergie, une particule libre se mouvant dans un champ de force nul, possède une vitesse constante mais rien n'est dit sur sa direction ni sur sa trajectoire, puisque l'énergie cinétique n'est pas une quantité vectorielle. La trajectoire peut donc être aussi bien rectiligne que curviligne sans que le principe de conservation de l'énergie ne soit violé. Cependant, le principe de moindre action exige que dans le cas présent la trajectoire soit rectiligne, conduisant au principe d'inertie de Newton. Le même raisonnement peut être appliqué à la conservation de l'impulsion et du moment cinétique, qui se déduisent également du principe de moindre action.

\section{Conclusion}

Cette première partie de l'article, portant sur le principe de moindre action, s'est attachée à en poser les fondements historiques tout en essayant de réduire au minimum le formalisme mathématique, assez élaboré, mais très largement traité dans de nombreux ouvrages. Le principe d'économie de Fermat, issu de l'optique géométrique, est clairement à l'origine de la réflexion entamée par Maupertuis aboutissant au principe de moindre quantité d'action, appliqué à un système mécanique. Ce principe consiste à minimiser la circulation de la quantité de mouvement d'un système dynamique pour en déterminer la trajectoire réelle. Euler et Lagrange ont affiné ce principe en le redéfinissant à travers l'échange optimal entre les deux réservoirs d'énergie qui caractérisent un système conservatif, à savoir les énergies cinétique et potentielle. L'élégance du traitement d'Euler-Lagrange, bien que complètement équivalent à la définition de Maupertuis, a pour conséquence de mieux faire ressortir l'analogie optique géométrique - mécanique, mise en lumière dans le dernier paragraphe de cette première partie et qui s'avèrera d'une incroyable fécondité. La deuxième partie traitera en effet de l'importance capitale de cette analogie qui sera l'œuvre de Hamilton et Jacob Jacobi, ouvrant la voie à l'avènement de la mécanique quantique. Le développement de la mécanique quantique repose sur trois piliers; (i) le quantum d'action découvert par Max Planck suivi du photon d'Einstein donnant à la lumière la dualité onde corpuscule, (ii) l'extension de cette dualité à la matière par Louis De Broglie, et enfin (iii) l'établissement par Erwin Schrödinger, sur la base du travail de Hamilton-Jacobi, de l'équation maîtresse de la mécanique quantique. La deuxième partie sera close par l'apport de Richard Feynman à l'électrodynamique quantique.

\section{RÉFÉRENCES}

[1] E. Klein, L'unité de la physique (Presse Universitaire de France, 2000)

[2] Il y au moins une exception au fait que la matière ne peut être que pesante, lui associant ainsi une masse. C'est l'existence du neutrino

[3] Citation de J.B. Conant extraite de l'ouvrage Introduction to concepts and theories in physical science, second edition, G. Holton, S.G. Brush (Princeton University Press, 1985), p. 195

[4] J.L. Basdevant, Le principe de moindre action et les principes variationnels en physique (Vuibert, 2010)

[5] Le principe de Fermat se transpose à un problème bien connu, celui du trajet que doit suivre un sauveteur, sur une plage puis dans l'eau, pour secourir un baigneur, sachant que la vitesse sur la plage est supérieure à celle dans l'eau. Le principe schématisé à la figure 3(b) aboutit à la loi $\sin \alpha / u_{1}=\sin \beta / u_{2}$

[6] J. Bernoulli, Bending of light rays in transparent non-uniform media and the Solution of the problem of determining the Brachistrochrone curve, Acta Eruditorum Lipsiae (1697)

[7] Plus tard, le terme « quantité » fut abandonné et le principe devient celui de « moindre action». 